\title{
Franz Fuchs et Tobias Daniels (dir.), Venedig und der oberdeutsche Buchmarkt um 1500
}

\section{Catherine Rideau-Kikuchi}

\section{OpenEdition}

\section{Journals}

Édition électronique

URL : http://journals.openedition.org/ifha/10591

DOI : 10.4000/ifha.10591

ISSN : 2198-8943

\section{Éditeur}

IFRA - Institut franco-allemand (sciences historiques et sociales)

\section{Référence électronique}

Catherine Rideau-Kikuchi, «Franz Fuchs et Tobias Daniels (dir.), Venedig und der oberdeutsche Buchmarkt um $1500 »$, Revue de l'IFHA [En ligne], Date de recension, mis en ligne le 27 février 2020, consulté le 24 septembre 2020. URL : http://journals.openedition.org/ifha/10591 ; DOI : https:// doi.org/10.4000/ifha.10591

Ce document a été généré automatiquement le 24 septembre 2020.

(C)IFHA 


\section{Franz Fuchs et Tobias Daniels (dir.), Venedig und der oberdeutsche Buchmarkt um 1500}

Catherine Rideau-Kikuchi

\section{RÉFÉRENCE}

Franz Fuchs et Tobias Daniels (dir.), Venedig und der oberdeutsche Buchmarkt um 1500, Wiesbaden : Harrassowitz Verlag, 2017, 136 p., 49,80€ 
Le numéro de 2017 du PirckheimerJahrbuch est révélateur de la richesse actuelle des travaux autour de l'histoire du livre et de son commerce. Les textes rassemblés ici sont issus d'un colloque qui s'est tenu à Venise les 26 et 27 novembre 2015. Comme le rappelle la préface, l'actualité scientifique était propice à cette discussion entre spécialistes de thèmes et d'origine variés, avec la traduction de la monographie d'Angela Nuovo, The Book Trade in Renaissance Italy, parue en 2013, et l'achèvement de la thèse de doctorat de Bettina Pfotenhauer sur les relations entre Nuremberg et Venise en 2014. Les communications ont donné lieu à la publication de ce volume autour d'une thématique générale, Venise et le marché

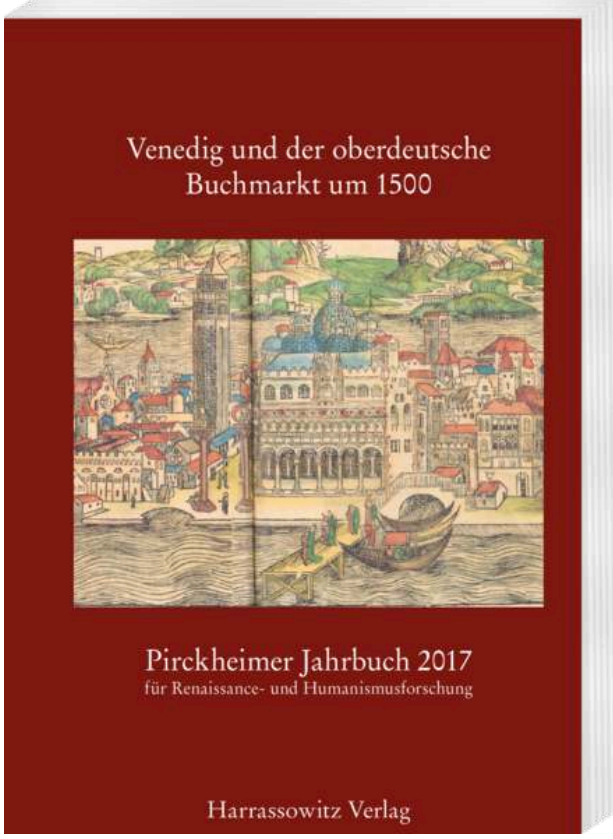
du livre en Allemagne du sud autour de 1500, abordée à partir d'approches différentes mais complémentaires.

«À la recherche des livres perdus. Deutsche Gelehrte in Venedig, 1821-1913» de Daniela Rando sert d'introduction aux travaux qui suivent, dans la mesure où l'article retrace l'histoire de la fascination des savants allemands pour Venise, et surtout pour ses archives et ses bibliothèques. L'autrice replace la tradition érudite allemande des Monumenta Germaniae Historica ou des Akademien der Wissenschaft de Vienne ou de Munich dans le contexte d'une attirance artistique, qui se manifeste notamment à travers les voyages littéraires du $\mathrm{XIX}^{\mathrm{e}}$ siècle. L'article retrace la généalogie des historiens et des études sur les sources présentes à Venise. Ces travaux, notamment ceux de Pertz Friedrich Thiesch sur la bibliothèque du cardinal Bessarion et de Henry Simonsfeld sur le Fondaco dei tedeschi, restent particulièrement utiles pour l'étude de l'imprimerie vénitienne et de ses liens avec le monde germanique.

Les deux contributions suivantes doivent se lire ensemble car elles participent des mêmes directions scientifiques. Angela Nuovo dresse un panorama d'ensemble des premiers temps de l'imprimerie et de la mise en place de son système de privilège. Si celui-ci se développe à la fin $\mathrm{du} \mathrm{XV}^{\mathrm{e}}$ siècle, les privilèges ne concernent qu'une petite partie des livres publiés (environ $14 \%$ entre 1527 et 1600). Ils sont pourtant essentiels pour l'activité économique de l'imprimerie. L'article d'Erika Squassina, approfondit la question des privilèges du point de vue de l'auteur. Les auteurs à qui les autorités attribuent un privilège ont des profils variés, mais ont bien souvent un lien privilégié avec Venise, qu'ils soient citoyens ou participent activement à la vie politique, intellectuelle ou culturelle de la cité. Les privilèges protègent la nouveauté pour favoriser le développement tant économique que culturel de la Sérénissime. La figure de l'auteur se construit donc largement en lien avec une volonté politique, comme Michèle Clément l'a bien montré dans le cas des privilèges vénitiens. On signale ici qu'Angela Nuovo dirige actuellement le projet ERC « Early Modern Book Trade » auquel Erika Squassina participe ; ce projet a notamment permis la conception et la mise en 
ligne d'une base de données recensant tous les privilèges vénitiens entre 1469 et 1545 (emobooktrade.unimi.it/db/public/frontend).

La contribution de Tobias Daniels apporte une lumière nouvelle sur les réseaux commerciaux de la principale compagnie d'imprimerie vénitienne avant 1480 . Grâce à un important travail archivistique à partir de documents vénitiens, milanais et de différentes villes allemandes, ainsi qu'une compilation de la - très vaste! bibliographie existante, l'auteur est en mesure de dessiner de façon très précise les modalités de commerce entre la compagnie vénitienne et le marché allemand, ainsi que leur chronologie. Grâce à des documents inédits, l'auteur souligne le rôle fondamental de Peter Ugelheimer, de son épouse puis veuve Margarita, et surtout de son beau-frère Loy Jostenhöffer resté à Francfort.

Cette approche archivistique est complétée de façon bienvenue par la contribution de Christoph Reske, qui utilise une méthodologie plus strictement liée à l'histoire du livre. Il retrace l'évolution des techniques d'impression musicale entre imprimeurs italiens et allemands, ce qui permet de relativiser la position souvent prédominante dans ces études d'Ottaviano Petrucci pour redonner leur place à d'autres imprimeurs qui ont inventé des solutions pour résoudre le problème posé par l'impression des portées et des notes.

La dernière contribution de Bettina Pfotenhauer permet enfin de replacer l'implication des patriciens allemands dans le contexte de leurs contacts économiques approfondis avec Venise, en particulier dans le cas de Nuremberg. L'étude approfondie des correspondances de certains patriciens humanistes de la ville permet de rendre compte de la circulation des livres vénitiens vers la ville, et du rôle de Nuremberg comme plaque tournante du commerce du livre, notamment humaniste.

À travers l'étude systématique des privilèges, l'analyse matérielle des livres, le croisement des sources de la pratique ou des correspondances, ces différentes contributions éclairent les premiers temps de l'imprimerie, et témoignent de l'importance des liens entre Venise et le monde germanique pour le développement de ces presses.

\section{INDEX}

Index chronologique : Moyen Âge, période moderne

Thèmes : Histoire de la culture, histoire économique

\section{AUTEURS}

CATHERINE RIDEAU-KIKUCHI

Service public de l'enseignement et de la recherche 